\title{
CURA ESPONTÂNEA DA LEISHMANIOSE CAUSADA POR LEISHMANIA VIANNLA BRAZILIENSIS EM LESÕES CUTÂNEAS
}

\author{
Jackson Maurício Lopes Costa, Kyola Costa Vale, Flávio França, Ana \\ Cristina R. Saldanha, Joilda Oliveira da Silva, Ednaldo L. Lago, Philip \\ D. Marsden, Albino V. Magalhães, Conceição de Maria P. e Silva, Artur \\ Serra Neto e Clóvis Eduardo S. Galvão
}

\begin{abstract}
Os autores relatam que durante 14 anos de trabalho clínico em campo, realizado nas comunidades de Trés Brafos e Corte de Pedra, Bahia, acompanharam 1.416 pacientes portadores de Leishmaniose Tegumentar Americana, cuja espécie envolvida na transmissão, é predominantemente a Leishmania Viannia brasilienses. A terapêutica utilizada rotineiramente nos casos éo antimoniato-N-metilglucamina (Glucantime). Contudo, 16 pacientes do sexo masculino recusaram-se a utilizar a medicação e 6 do sexo feminino encontravam-se em periodo gestacional, portanto näo utilizaram o medicamento. Estes pacientes foram acompanhados por um periodo entre 4 a 12 anos, a partir do diagnóstico. Observou-se que em 9 pacientes (40,9\%) desta casuistica, o tempo de cicatrizaçâo após o aparecimento da lesão, pode ser calculado em 6 meses de evolucáa. Quando se eleva a observação para 12 meses, temos que 19 pacientes $(86,3 \%$ ) cicatrizaram suas lesões neste periodo. Em 3 casos (13,6\%) as lesöes permaneceram ativas por mais de 12 meses. Conclui-se que os determinantes da cicatrizaçáo natural das lesóes produzidas por Leishmania Viannia braziliensis permanecem desconhecidos, dificultando para nós entendermos e compararmos aos efeitos das drogas utilizadas no tratamento da leishmaniose tegumentar.
\end{abstract}

Palaras-chaves: Cura espontanea. Leishmania Viannia braziliensis. Lesäo cutanea.

Segundo Pessoa 15.1941 . as curas espontâneas das lesões cutâneas na Leishmaniose Tegumentar Americana se processam em número bastante acentuado de casos, apresentando uma certa semelhança ao botão do oriente.

Raol6. 1911. refere que após a ulceração Leishmaniótica constituida, caso não haja infecção secundária, a tendência da lesão é a cicatrização espontânea. Nota-se, portanto, que apesar das diversidades clinicas, a Leishmaniose Tegumentar Americana apresenta, em alguns casos, caráter extremamente benigno, curando-se com simples medicações tópicas 418 .

A leishmaniose causada por Leishmania Viannia braziliensis que apresenta geralmente lesões destrutivas na pele, atingindo também as mucosas, em alguns casos cura-se espontaneamente 51418 . Guer$\mathrm{ra}^{6}, 1983$, estudando pacientes provenientes da região de Três Braços-Bahia, área reconhecidamente de Leishmania Viannia braziliensis, observou 10 paci-

Núcleo de Medicina Tropical e Nutrição da Universidade de Brasilia. Superintendencia de Campanhas (SUCAM) Ministério da Saude. Brasilia. DF

Suporte financeiro do USPITS Grant AI 16282-04.

Endereço para correspondencia: Dr. Jackson M.L. Costa. Disc. Doenças Infecciosas e Parasitárias/Depto. de Patologia/FM/UFMA. Largo dos Amores 351.65000 São Luis. MA.

Recebido para publicação em 28/01/88. entes. todos com lesōes cutâneas, que se curaram espontaneamente. Recentemente, Marsden ${ }^{14}$, 1986, observando pacientes da mesma região, portadores de comprometimento mucoso, refere 2 casos com ulceração no septo nasal que tiveram cura espontânea.

Costa ${ }^{3}, 1986$, ao estudar um surto epidêmico da doença na região de Corte de Pedra, município de Valença observou 4 casos, 1 do sexo masculino que se recusou em usar a medicação e 3 do sexo feminino que se encontravam em periodo gestacional, todos portadores de lesōes cutâneas, que curaram espontaneamente.

Tais fatos, motivaram-nos a fazer um estudo retrospectivo dos casos que se curaram espontaneamente, observados durante um período de 14 anos por nossa equipe nas regiōes de Três Braços e Corte de Pedra-Bahia, os quais serão relatados no presente trabalho.

\section{MATERIAL E MÉTODOS}

A amostra estudada, 22 pacientes, representa $1.6 \%$ da nossa casuística total, todos oriundos das áreas endêmicas de Três Braços e Corte de PedraBahia, regiōes pertencentes aos municípios de Wenceslau Guimarāes, Cravolândia, Ubaíra e Valença. Localidades onde ainda restam pequenas áreas antigas de Mata Atlântica, porem em grande parte ocupadas pelas culturas do cacau, cravo-da-índia e mandioca, sendo a topografia da região bastante acidenta$\mathrm{da}^{3} 813$.

Nessas áreas, o agente etiológico predominante e a Leishmania Viannia braziliensis e o principal 
Costa JML, Vale KC, França F, Saldanha ACR, Silva JO, Lago EL, Marsden PD, Magalhães AV, Silva CMP, Serra Neto A, Galväo CES. Cura espontânea da leishmaniose causada por Leishmania Viannia braziliensis em lesões cutâneas. Revista da Sociedade Brasileira de Medicina Tropical 23: 205-208, out-dez, 1990.

transmissor da doença é o Lutzomyia whitmani ${ }^{14}$. Até maio de $1989,1.416$ pacientes leishmanióticos foram estudados nas diversas formas clínicas, sendo que $22(1,6 \%)$ da casuística total evoluiram para cura espontânea: $16(72,7 \%$ ) recusaram a utilização da medicação por algum motivo, enquanto que 6 mulheres $(27,3 \%)$ não foram tratadas por se encontrarem em período gestacional.

$\mathrm{O}$ diagnóstico dos pacientes baseou-se nos aspectos clínicos das lesões, exames imunológicos (Intradermorreação de Montenegro e Imunofluorescência Indireta) e parasitológicos (esfregaço, cultura em meios artificiais e histopatologia). Utilizamos como critério de cura, a cicatrização completa das lesões, sem sinais de reativação das mesmas.

\section{RESULTADOS}

Os dados clínicos dos pacientes revelaram que a idade dos mesmos foi variável de 3 a 50 anos. Clinicamente, havia predomínio de lesão ulcerada em 21 casos $(95,5 \%)$ e apenas 1 paciente apresentava lesão pápulo eritematosa.
Entre os exames realizados destacamos: esfregaço das bordas da lesão em 11 casos $(50 \%)$, com 4 $(36,3 \%)$ positivos; cultura em meios artificiais em 6 casos $(27,2 \%)$, sendo apenas 1 positivo, onde se identificou o agente como pertencente ao complexo brasiliensis, caracterizado, posteriormente, pela técnica de anticorpos monoclonais como sendo Leishmania Viannia brasiliensis.

Dos 22 casos, em $9(40,9 \%)$ o tempo de cicatrização após o aparecimento da lesão pode ser calculado em 6 meses de evolução. Quando se eleva para 12 meses de observação tivemos 19 pacientes $(86,3 \%)$ que cicatrizaram suas lesões neste periodo. Em 3 casos $(13,6 \%)$ as lesões permaneceram ativas por mais de 12 meses. A Tabela I, traz detalhes dos aspectos clínicos, laboratoriais e evolutivos dos pacientes estudados.

Apenas 1 caso apresentou recidiva de lesão (LTB0227), o qual foi posteriormente tratado com glucantime $15 \mathrm{mg} / \mathrm{sb} / \mathrm{kg} /$ peso, em 2 séries de 10 dias cada, com intervalos de 10 dias de uma série para outra, não exibindo, até o presente momento comprometimento mucoso.

Tabela 1 - Caracteristicas clinicas, laboratoriais e evolutivas dos 22 pacientes curados espontaneamente.

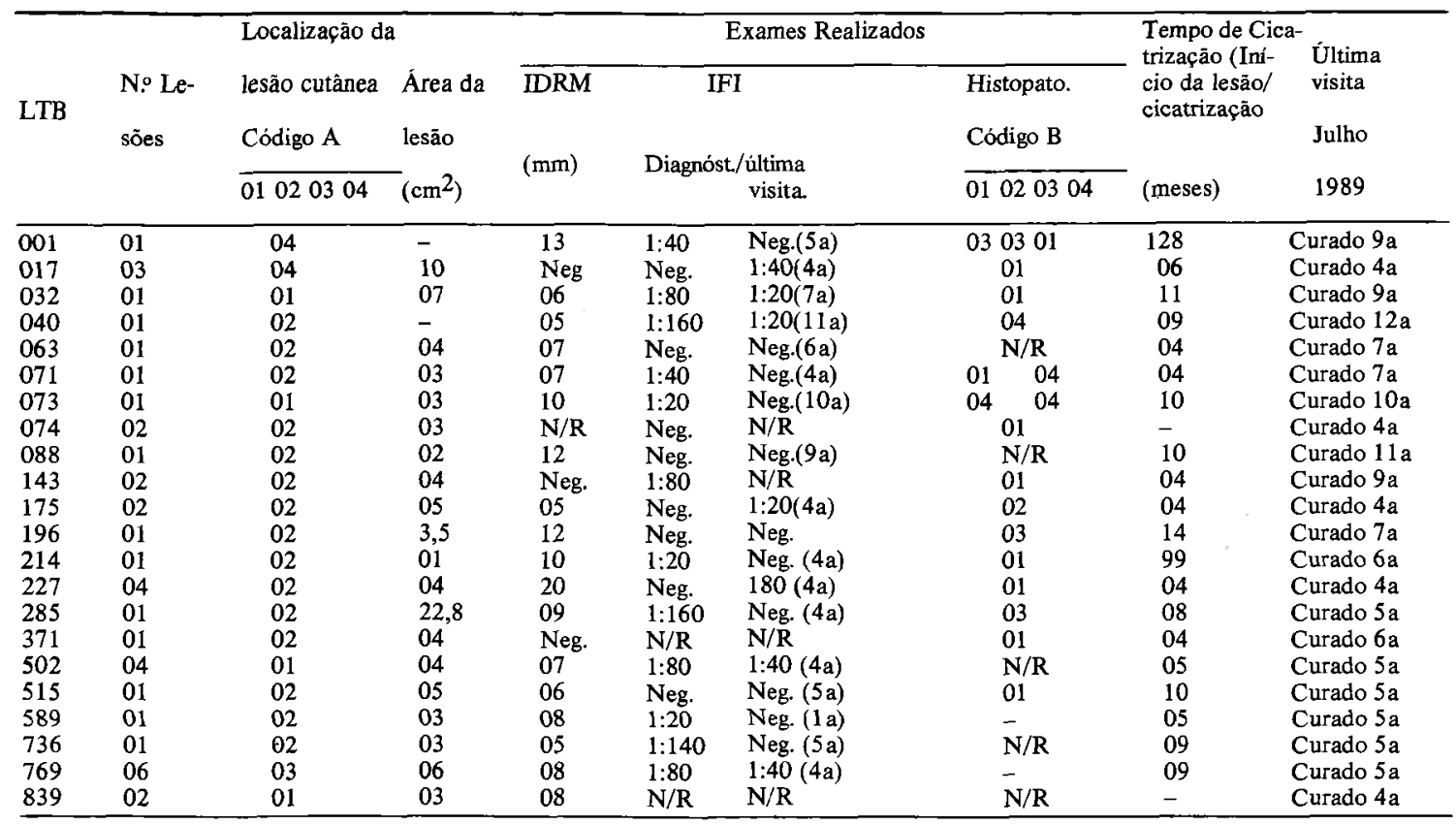

Chave Localização (Código A) 01) MMSS (Membros Superiores)

02) MMII (Membros Inferiores)

03) MMSS e MMII (Membros Superiores e Membros Inferiores)

04) MMII e Glúteo (Membros Inferiores e Glúteo)

(Código B) 01) Padrão de reação exsudativa celular exclusiva (celularidade mista)

02) Padrāo de reação exsudativa e necrótica

03) Padrão de reação exsudativa e necrótica granulomatosa

04) Padrão de reação exsudativa e granulomatosa

LTB $=$ Leishmaniose Três Braços 
Costa JML, Vale KC, França F, Saldanha ACR, Silva JO, Lago EL, Marsden PD, Magalhàes AV, Silva CMP, Serra Neto A, Galvão CES. Cura espontânea da leishmaniose causada por Leishmania Viannia braziliensis em lesóes cutâneas. Revista da Sociedade Brasileira de Medicina Tropical 23: 205-208, out-dez, 1990.

\section{DISCUSSÃO}

Os dados mostrados neste trabalho confirmam que a cura espontânea na Leishmaniose Tegumentar Americana causada por Leishmania Viannia braziliensis pode ocorrer, devendo ser o fenômeno mais freqüente entre portadores de lesões cutâneas produzidas pelas leishmanioses, nas áreas endêmicas, ao contrário do que normalmente se pensa. Deduz-se que muitos pacientes com lesões evolutivas para cura rápida e outros que desenvolvem uma infecção leishmaniótica sem lesões típicas, podem não procurar cuidados médicos.

Recentemente, Barral e cols ${ }^{2}$ isolaram Leishmania brasiliensis dos linfonodos de pacientes com linfoadenomegalia inguinal sem qualquer lesão tegumentar ou evidência de Leishmaniose visceral clássica. A predôminancia de infecções subclinicas sobre a doença na área endêmica é observada na Leishmaniose visceral ${ }^{1}$, de forma semelhante a várias outras infecções.

E dificil explicar a grande variação nos prazos de cura espontânea em diferentes pacientes, como se pode observar neste trabalho. Heinzel e cols7, em trabalho experimental, observaram que o curso da Leishmaniose tegumentar em camundongos é bastante diversificado, havendo dependência na variação do patrimônio genético, existindo camundongos parcialmente resistentes, os quais evoluem para cura espontânea e outros extremamente suscetiveis que apresentam uma doença de curso fatal após disseminação parasitária por todo o corpo.

Nestes animais a diferença do curso da doença tem sido atribuída a diversidade da resposta imune celular aos antigenos de leishmania. Os camundongos resistentes, após infecção pela leishmania, apresentam uma ativação de células $T h_{1}$ e nos seus linfonodos de drenagem da lesão observa-se uma predominância dos RNAm para IL 2 e interferon gamma; nos camundongos susceptíveis a ativação de célula $\mathrm{Th}_{2}$ é acompanhada de uma predominância de RNAm IL-4.

Em humanos portadores de Leishmaniose Tegumentar Americana, os estudos histopatológicos de Riddley e cols ${ }^{17}$ e Magalhães e cols ${ }^{9} 10$, sugerem que a Leishmania Viannia braziliensis induz não somente uma resposta imune celular local, revelada por hipersensibilidade tardia contra antigenos de leishmania, que aparecem de 10 dias a 6 semanas após o início dos sintomas, como também, o aparecimento de graus variáveis de necrose e formação de granulomas, podendo haver mudança entre os mesmos durante a evolução da lesão. Estas oscilações no processo de necrose e formação de granulomas é que dificultam a formação de uma base prognóstica no quadro histopatológico da lesão 9101112 .

\section{SUMMARY}

In field clinics in the comunities of Três Braços and Corte de Pedra, Bahia, we have attended 1.416 patients with tegumentary leishmaniasis in fourteen years, the predomi- nant species in transmission is Leishmania Viannia brasi liensis $(L V B)$. Because of the danger of metastasis with this infection treatment was routinely recomended with Glucantime. However sixteen patients refused injection therapy and six women were pregnant when seen and not treated. All patients were followed up in our clinic. All these patients closed their skin ulcers although one subsequently relapsed. Patients were followed up for variable periods (four to twelve years), after the diagnosis. In nine patients $(40,9 \%$ ) of the cohort, the time to healing after initiation of the lesion was calculated as six months of evolution. At twelve months, nineteen patients $(86,3 \%)$ had complete healing of their lesions. In three patients an active lesion was present for longer than one year. The determinants of this variable natural evolution of human $L V B$ lesion remains completely unknown. It is difficult for us to understand and compare the effects of therapeutic agents in mucocutaneous leishmaniasis.

Key-words: Spontaneous healing. Leishmania Viannia braziliensis. Cutaneous lesion.

\section{REFERÊNCIAS BIBLIOGRÁFICAS}

1. Badaró R, Jones TC, Carvalho EM, Sampaio D, Reed SG, Barral A, Teixeira $R$ e Johnson JR WD. New perspectives on a subclinical form of visceral leishmaniasis. The Journal of Infectious Disease 154: 10031011, 1986.

2. Barral A, Carvalho EM, Grimaldi G, Almeida RP, Ribeiro de Jesus A, Netto EM, Santos IA, Badaró R, Barral Netto M Johnson JR WD. Clinical and diagnostic relevance of lynphadenopathy in human cutaneous leishmaniasis (Em via de publicação).

3. Costa JML. Estudo clínico-epidemiológico de um surto epidêmico de Leishmania Tegumentar Americana em Corte de Pedra-Bahia. Tese de Mestrado, Universidade de Brasília, Brasília, 1986.

4. Costa JML, Aristimino Barrios L, Netto EM, Marsden PD. Topical Pentostam in an Attempt to produce more rapid healing of skin ulcers due to Leishmaniabrasiliensis brasiliensis. Revista da Sociedade Brasileira de Medicina Tropical 19: 199-200, 1986.

5. Costa JML, Netto EM, Vale KC, Osaki NK, Tada MS, Marsden PD. Spontaneous healing of Leishmania brasiliensis brasiliensis skin ulcers. Transations of the Royal Society of Tropical Medicine and Hygiene 21: $606,1987$.

6. Guerra MVF. Avaliaçāo da quimioterapia na leishmaniose muco-cutânea em Três Braços-Bahia. Documento mimiografado.

7. Heinzel FP, Sadick MD, Holaday BJ, Coffman RL e Locksley RM. Reciprocal expression of interferon gamma or interleukin-4 during the resolution or distinat helper T cell subsets. Journal of Experimental Medicine 169: 59-72, 1989.

8. Llanos-Cuentas EA. Estudo clínico evolutivo da Leishmaniose em área endêmica de Leishmania brasiliensis brasiliensis, Três Braços, Bahia. Tese de Mestrado, Universidade de Brasília, Brasília, 1984.

9. Magalhães AV, Moraes MAP, Raick NA, Llanos Cuentas EA, Costa JML, Cuba Cuba CA, Marsden PD. Histopatologia da Leishmaniose Tegumentar por Leishmania brasiliensis brasiliensis. I. Padrões histo patológicos e estudo evolutivo das lesōes. Revista do 
Costa JML, Vale KC, França F, Saldanha ACR, Silva JO, Lago EL, Marsden PD, Magalhães AV, Silva CMP, Serra Neto A, Galvão CES. Cura espontânea da leishmaniose causada por Leishmania Viannia braziliensis em lesões cutâneas. Revista da Sociedade Brasileira de Medicina Tropical 23: 205-208, out-dez, 1990.

Instituto de Medicina Tropical de São Paulo 28: 253$262,1986$.

10. Magalhães AV, Moraes MAP, Raick NA, LlanosCuentas EA, Costa JML, Cuba Cuba CA, Marsden PD. Histopatologia da Leishmaniose Tegumentar por Leishmania brasiliensis brasiliensis. II. Resposta humoral tissular. Revista do Instituto de Medicina Tropical de São Paulo 28: 293-299, 1986.

11. Magalhāes AV, Moraes MAP, Raick NA, LlanosCuentas EA, Costa JML, Cuba Cuba CA, Marsden PD. Histopatologia da Leishmaniose Tegumentar por Leishmania brasiliensis brasiliensis. III. Reação celular nos tecidos. Revista do Instituto de Medicina Tropical de SãoPaulo 28: 300-311, 1986.

12. Magalhães AV, Moraes MAP, Raick NA, LlanosCuentas EA, Costa JML, Cuba Cuba CA, Marsden PD. Histopatologia da Leishmaniose Tegumentar por Leishmania brasiliensis brasiliensis. IV. Classificação histopatológica. Revista do Instituto de Medicina Tropical de São Paulo 28: 421-430, 1986.
13. Marsden PD. Novos conceitos sobre os antimoniais pentavalentes no tratamento da Leishmaniose. Revista da Sociedade Brasileira de Medicina Tropical 16: 172$174,1983$.

14. Marsden PD. Mucosal leishmaniasis ("Espundia" Escomel 1911). Transactions of the Royal Society of Tropical Medicine and Hygiene, 80: 859-875, 1986.

15. Pessoa SB. Dados sobre a epidemiologia da Leishmaniose Tegumentar em São Paulo. O Hospital 19: 385 $409,1941$.

16. Raó CA. Leishmaniose ulcerosa e seu parasita. Tese, Rio de Janeiro, 1911.

17. Ridley DS, Marsden PD, Cuba CC, Barreto AC. A histopatology classification of mucocutaneous leishmaniasis in Brazil and its clinical evaluation. Transaction of the Royal Society of Tropical Medicine and Higiene 74: 508-514, 1980.

18. Solano AE, Hidalgo HH, Zeledon RA. Tratamento intralesional exitoso de la leishmaniasis por Leishmania brasiliensis panamensis com Glucantime. Medicina Cutis ILA 12: 19-24, 1984. 